\title{
SURFACE STATES ON CLEAN Ge(111) SURFACES
}

\author{
H.J.W. ZANDVLIET and A. VAN SILFHOUT \\ Faculty of Applied Physics, University of Twente, P.O. Box 217, 7500 AE Enschede, The Netherlands
}

Received 13 July 1987; accepted for publication 27 October 1987

Surface states on $\mathrm{Ge}(111) \mathrm{c}(2 \times 8)$ are detected using ellipsometry, field effect and surface conductivity measurements. Optical transitions at 1.7, 1.9, 2.3, and 3.0 eV are observed. Surface states in the gap are detected with DC field effect measurements. We found a relationship between the surface conductivity and the ellipsometric parameter $\psi$ at the optical transitions during oxygen adsorption. A maximum in the surface conductivity is found for a coverage of 0.15 monolayer oxygen, at the same point Surnev detected (with work function measurements) a change of the elementary dipole moment.

\section{Introduction}

The electronic structure of group IV semiconductor surfaces has been the subject of numerous studies, however, most of these studies are devoted to silicon and not to germanium surfaces. This subject has been and continues to be a controversial subject $[1,2]$. Recently, surface state distributions have been measured directly by angle-resolved photoemission experiments [3-5]. These authors observed in their emission spectra at 0.8 and $1.4 \mathrm{eV}$ bindingenergy features (referred to the Fermi level) for $\operatorname{Ge}(111) \mathrm{c}(2 \times 8)$ surfaces. Optical measurements, which detect optical transitions from filled to unoccupied surface state bands, are reported by Meyer et al. [6-10] and by Nannarone et al. [11] for $\mathrm{Ge}(111) \mathrm{c}(2 \times 8)$ and $\mathrm{Ge}(111) 2 \times 1$ surfaces. The last authors used optical reflectometry and the first one spectroscopic ellipsometry. Despite all these measurements the surface state distribution on $\mathrm{Ge}(111)$ is still far from being understood.

In this papcr we investigate ellipsometric and electrical measurements of clean and oxidized Ge(111) surfaces. Optical measurements give both information on the thickness and the complex dielectric constant of the adsorbed layer on Ge surfaces and about optical transitions from filled to empty electron energy bands [12]. From our measurements the existence of surface states both in, and outside, the gap are shown. The combination of spectroscopic el- 
lipsometry and electrical measurements makes it possible to follow "in situ" and nondestructively the adsorption process of oxygen on $\mathrm{Ge}(111)$ surfaces.

\section{Experimental}

The experiment: vere performed in a stainless steel UHV system equipped with a single-pass cylindrical mirror analyzer (Riber CMA OPC 105) for Auger electron spectroscopy, a quadrupole mass spectrometer (Riber QMM 16 quadrupole), a spectroscopic PSA(rot) ellipsometer and an arrangement for field effect and surface conductance measurements. The UHV system is pumped by a turbomolecular pump (Pfeiffer TSU 200) and a $400 \mathrm{l} / \mathrm{s}$ ion-getter pump integrated with a titanium sublimator (Riber UNI-7). The base pressure obtained was $1 \times 10^{-10}$ Torr. A detailed description of the measuring system is given in ref. [13j.

The Ge(111) samples, dimensions $30 \times 10 \times 0.3 \mathrm{~mm}^{3}$ and resistivity $23 \Omega$ cm p-type, were polished mirror-like and cleaned prior to deposition of the electrical contacts. For the conductivity measurements four titanium contacts were evaporated on the samp'e through a tantalum mask [14,15]. The cleaning procedure for obtaining a clean $\mathrm{Ge}(111) \mathrm{c}(2 \times 8)$ consisted of several cycles of $\mathrm{Ar}^{+}$bombardment $\left(45^{\circ}, 800 \mathrm{eV}, 2 \mu \mathrm{A} \mathrm{cm}{ }^{-2}\right)$ and annealing at $850 \mathrm{~K}$ $[3,4,16-18]$. Following this procedure, the intensity of the carbon Auger peak was within the noise level of the Auger spectrometer. For field effect measurements a chopped constant current was sent through the outer pair of contacts of the sample, while the resulting potential was measured between the inner pair of contacts. In this way nonlinear behaviour of the metal-semiconductor contact is eliminated. Use of an alternating current averages out any thermoor photovoltage resulting from a nonuniform sample temperature or illumination [15].

The field-electrode consisted of a Si plate $\left(8 \times 8 \mathrm{~mm}^{2}\right)$ with a $\mathrm{SiO}_{2}$ toplayer which could be placed in front of the sample. Typical values for the capacitance between sample and field-electrode are about $10-100 \mathrm{pF} \mathrm{cm} \mathrm{cm}^{-2}$. The parallelism of this assembly with respect to the sample surface was adjusted by three springs located at positions around the sample.

The ellipsometric measurements were performed with a rotating analyzer in the photon-energy range from 1.5 to $3.5 \mathrm{eV}$ as described in ref. [13]. The ellipsometric quantities $\Delta$ and $\psi(\rho=\tan \psi \exp \Delta)$ were determined by the two-zone method. We have used a teirasil window which is transparant for wavelengths in the region of $150 \mathrm{~nm}$ to $3 \mu \mathrm{m}$. Typical window corrections are negligible in $\psi$ and small for $\Delta$, in the worst case $0.3^{\circ}$ [13]. The experimental errors in $\delta \Delta$ and $\delta \psi$ are $0.02^{\circ}$ a.d $0.01^{\circ}$ respectively, corresponding to an error in the magnitude of the $\operatorname{Im}\left(\epsilon_{\mathrm{ss}}-\epsilon_{\mathrm{b}}\right)$ curve of about 1 [9]. 
The Auger spectra were recorded using a primary beam energy of $2 \mathrm{keV}$, primary current density of $\sim 0.5 \mu \mathrm{A} \mathrm{cm}^{-2}$, modulation voltage of the energy analyser $3 \mathrm{~V}_{\mathrm{pp}}$, a sweep rate of $1 \mathrm{eV} / \mathrm{s}$ and a lock-in time constant $<1 \mathrm{~s}$.

\section{Results}

3.1. Electronic structure of the clean Ge(111) surface

Field effect measurements indicate the presence of a p-type surface layer on the cleaned Ge samples (see fig. 1), in agreement with literature data [19-24].

The absolute value of the field effect mobility $\mu_{\mathrm{fe}}$, defiried as

$\mu_{\mathrm{fe}}=-\mathrm{do} / \mathrm{d} Q_{\text {ind }}$,

where $\sigma_{s}$ is the surface conductivity and $Q_{\text {ind }}$ the total induced charge per unit area, is about $-10 \mathrm{~cm}^{2} / \mathrm{V} \cdot \mathrm{s}$ for our cleaned crystals. The field effect is given by $Q_{\text {ind }}$ and the relative change of conductivity $\Delta G / G$ (see fig. 1), e.g. for $V=1000 \mathrm{~V}, Q_{\text {ind }}$ is $1.4 \times 10^{-8} \mathrm{C} / \mathrm{cm}^{2}$ and $\Delta \sigma_{\mathrm{s}}$ is $1.4 \times 10^{-7} \Omega^{-1}$.

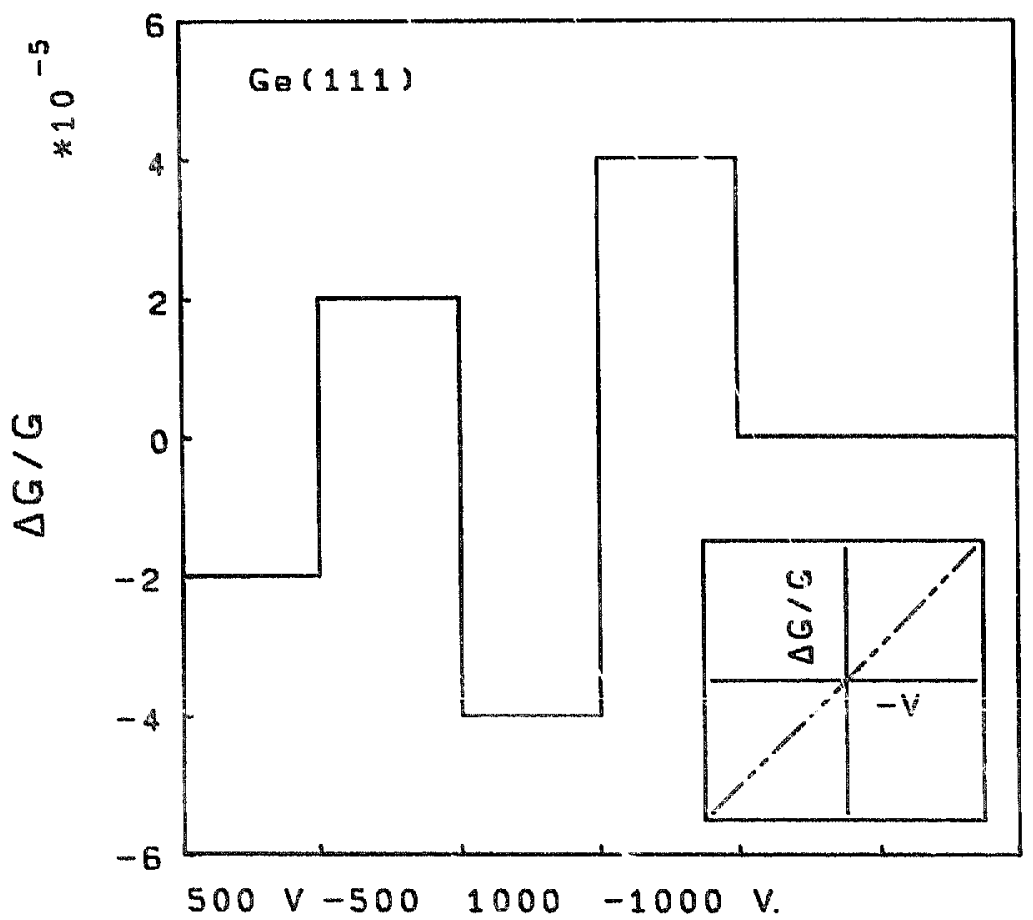

Fig. 1. Relative change $\Delta G / G$ of the conductivity at values of $V=V_{\text {electrode }}-V_{\text {semiconductor }}=0$, $500,-500,1000,-1000$ V. $C=14 \mathrm{pF} / \mathrm{cm}^{2}$. 
The semiconductor plate is connected to earth and after the application of a potential difference $V$, the charge $Q_{\text {ind }}$ on the semiconductor surface is equal to $C V$, where $C$ is the capacitance. We can write:

$\mathrm{d} Q_{\text {ind }}=C V=\mathrm{d} Q_{\mathrm{ss}}+\mathrm{d} Q_{\mathrm{sc}}$,

where $Q_{\mathrm{ss}}$ is the amount of charge, located in surface states, and $Q_{\mathrm{sc}}$ is that located in the space charge.

Assuming $k$ types of acceptor states, state density of the $i$ th state $N_{\mathrm{sa} i}$, Fermi factor $f_{\mathrm{sa} i}$ and $r$ types of donor states, state density of the $j$ th state $N_{\mathrm{sd} j}$ and Fermi factor $f_{\mathrm{sd} j}$, then $\mathrm{d} Q_{\mathrm{ss}}$, the change of the charge in the surface states as a result of the application of the transverse field, is given by:

$\mathrm{d} Q_{\mathrm{ss}}=-e^{2} \mathrm{~d} V_{\mathrm{s}} / k T\left[\sum N_{\mathrm{sa} i} f_{\mathrm{sai}}\left(1-f_{\mathrm{sai} i}\right)+\sum N_{\mathrm{sd} j} f_{\mathrm{sd} j}\left(1-f_{\mathrm{sd} j}\right)\right]$,

where $\mathrm{d} V_{\mathrm{s}}$, the change in band bending, is immediately given by the field effect $[20,24]\left(\mathrm{d} Q_{\mathrm{ss}} \simeq 1.4 \times 10^{-8} \mathrm{C} / \mathrm{cm}^{2}\right.$ and $\left.\mathrm{d} V_{\mathrm{s}}=3.2 \times 10^{-5} \mathrm{~V}\right)$. The term in square brackets can be calculated and has a value of about $7 \times 10^{13}$ per $\mathrm{cm}^{2}$ in good agreement with Boonstra $[19,24]$. The only statement that can be made is that, when plotting $f_{\mathrm{sa}, s d}\left(1-f_{\mathrm{sa}, s d}\right)$ against $\left(E_{\mathrm{F}}-E_{\mathrm{sa}, \mathrm{sd}}\right)$, there is a maximum of $1 / 4$ at $E_{\mathrm{F}}=E_{\text {sa,sd. }}$. Therefore, the value of the bracketed part of eq. (3) is only an indication of the minimum value of $\sum N_{\mathrm{sa}}+\sum N_{\mathrm{sd}}$. This minimum has a value of about $3 \times 10^{14}$ per $\mathrm{cm}^{2}$.

The $\mathrm{d} Q_{\mathrm{ss}}$ versus $\mathrm{d} V_{\mathrm{s}}$ curve appears to be a straight line for both positive and negative values of these parameters. This gives an a posteriori argument in favour of the statement that the charge carriers in the surface states are immobilized [20], so measured changes in the the surface conductivity can only be attributed to space charge effects.

By definition the surface conductivity $\sigma_{\mathrm{s}}$ is given by

$\sigma_{\mathrm{s}}=e\left(\mu_{\mathrm{ns}} \Delta N+\mu_{\mathrm{ps}} \Delta P\right)$,

where $e$ is the elementary charge, $\mu_{\mathrm{ns}}$ and $\mu_{\mathrm{ps}}$ are the effective carrier mobilities at the surface and $\Delta N, \Delta P$ the excess electron and hole concentrations per unit surface area [20-22]. The values of $\sigma_{\mathrm{s}} /\left(\sigma_{\mathrm{b}} d\right)$ can be calculated as a function of the band bending at the surface $V_{s}$, where $d$ is the sample thickness and $\sigma_{4}$ the bulk conductivity. The results of these calculations are shown in fig. 2 for intrinsic and $23 \Omega \mathrm{cm}$ p-type Ge at $300 \mathrm{~K}$; for a p-type surface there is no great difference between intrinsic and p-type Ge, so intrinsic formulas can be used without large errors.

If at room temperature and at a constant oxygen pressure $\left(2 \times 10^{-5}\right.$ torr), molecular oxygen was added, the p-type surface conductivity first increased then decreased after increased oxygen exposure to a saturation value (fig. 3 ). Oxygen exposures of a few Torr seconds give a somewhat lower value. If we assume that the saturation value corresponds to the flat band situation (this is not completely correct see e.g. ref. [19]) we can calculate the band bending at 


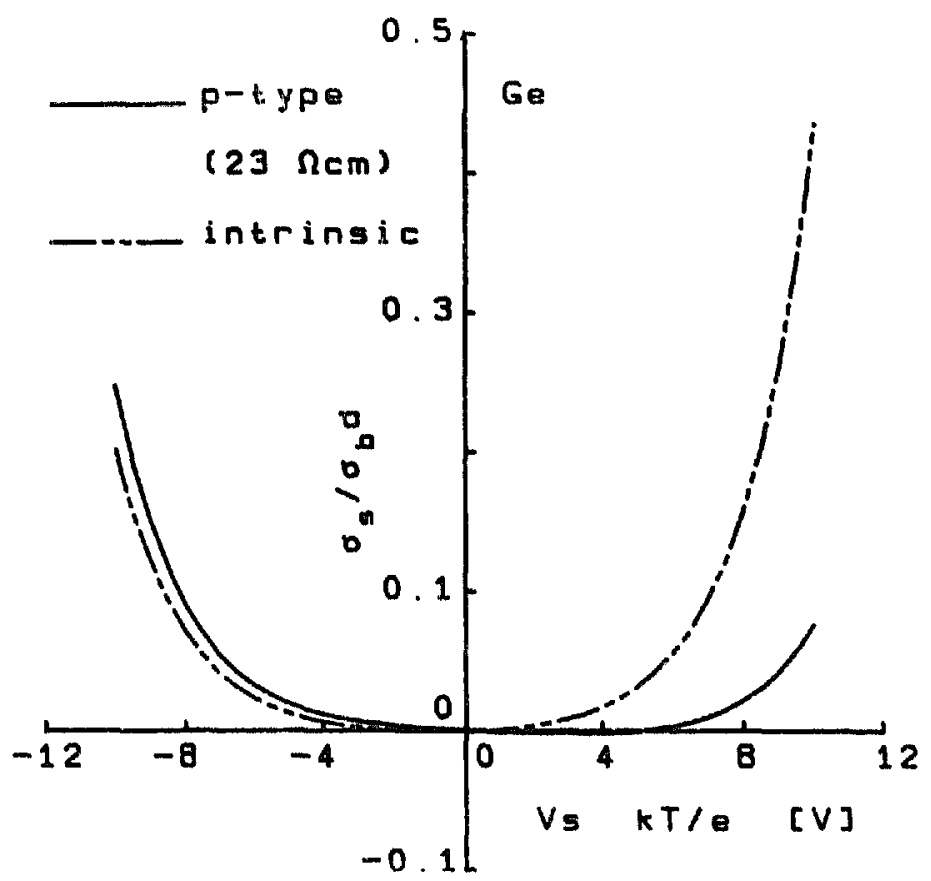

Fig. 2. Calculated curves of $\sigma_{\mathrm{s}} / \sigma_{\mathrm{b}} d$ versus band bending at the surface for intrirsic and p-type Ge.

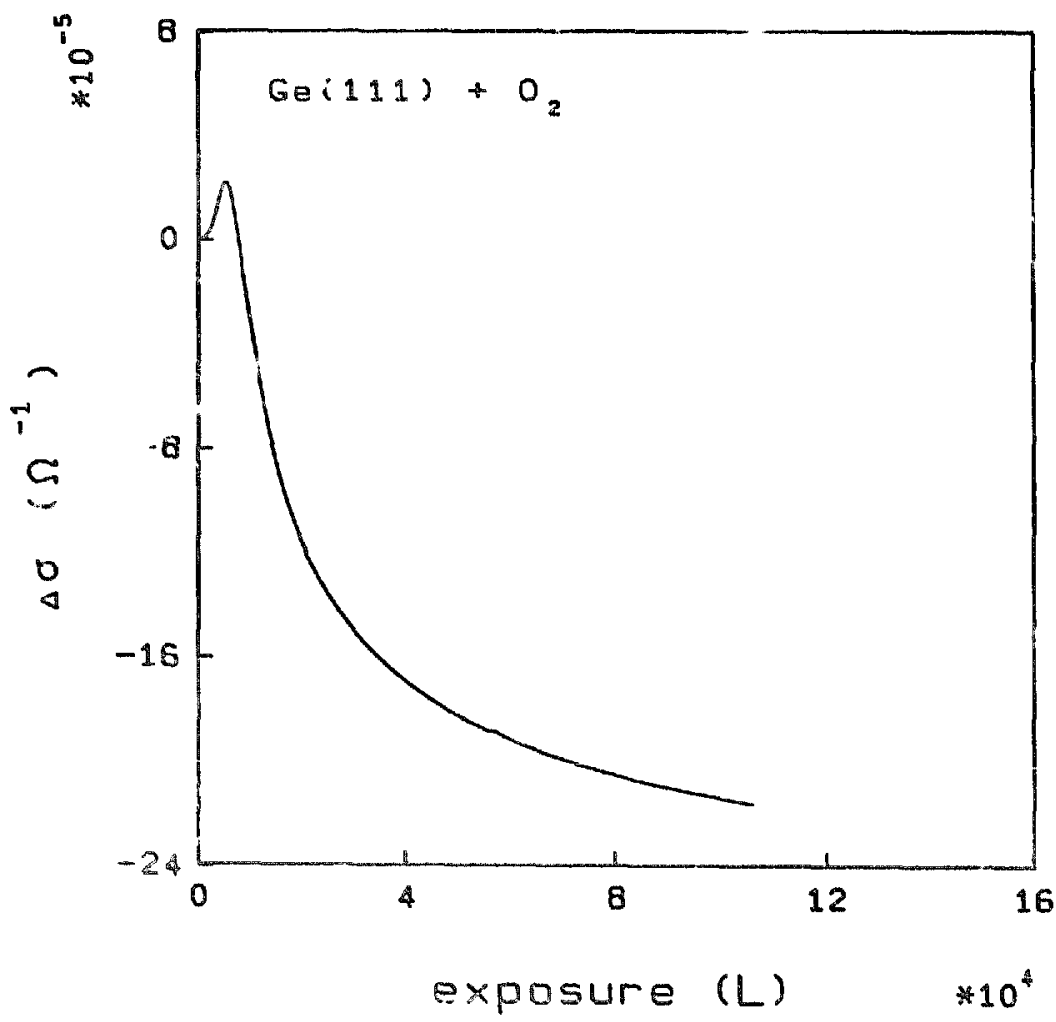

Fig. 3. Conductivity changes of the Ge sample versus oxygen exposure. 


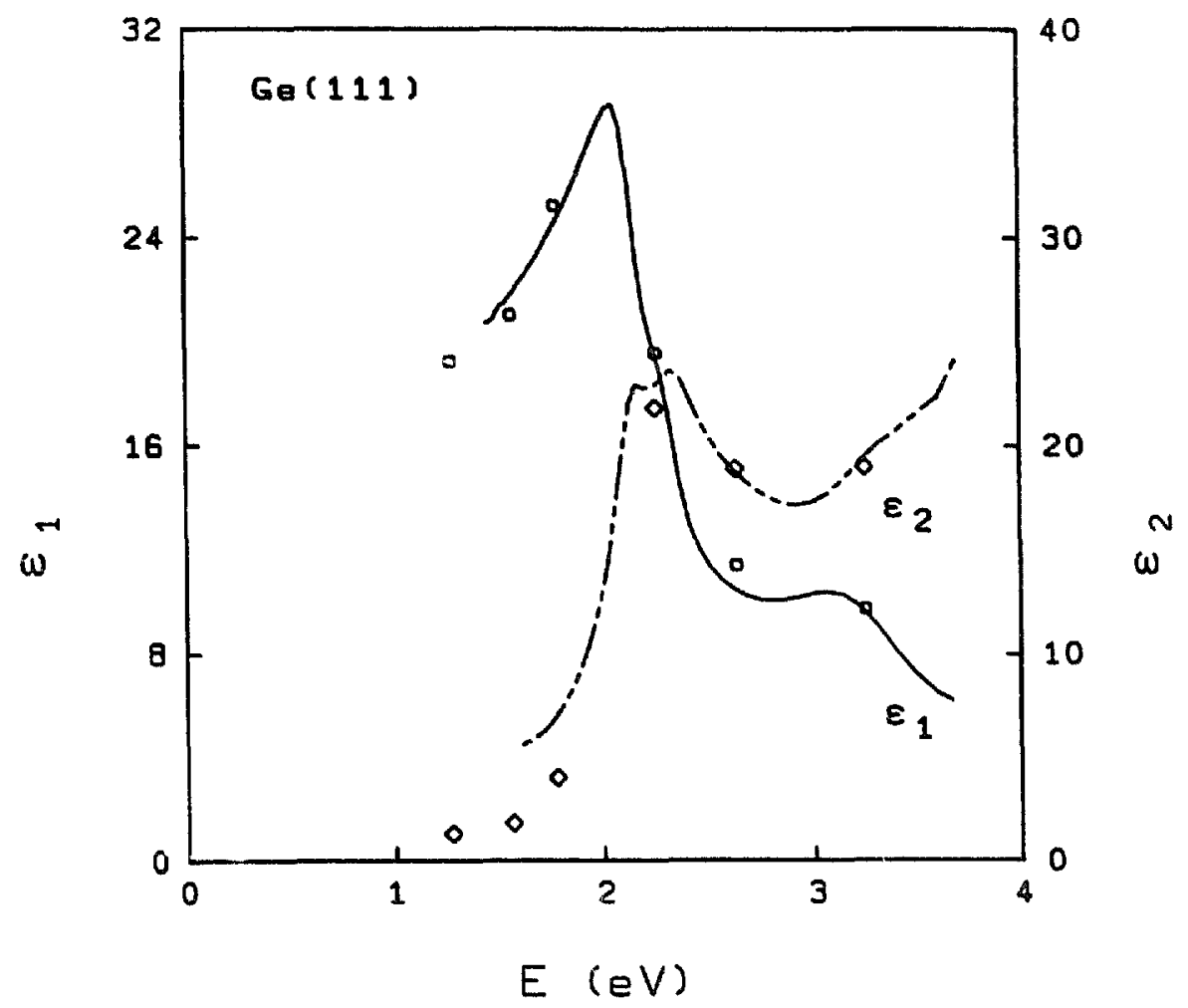

Fig. 4. Dielectric function data for c-Ge(111) (continued curves, this work) compared with Meyer et al. [6] (discrete points).

the cleaned Ge surface. The surface is considered to be nondegenerate, no fast holes and fast electrons are assume,$\Delta N$ is neglected, the sample is considered intrinsic, and $\mu_{\mathrm{ps}}$ is replaced by the known value $\mu_{\mathrm{p}}$. The last assumption introduces errors of about $20 \%$, see for example refs. [21,25]. Then it foliows that the value is at least $-280 \mathrm{mV}$ (uncertainty about $10 \mathrm{mV}$ ), in good accordance with Boonstra et al. [19,23,24], Gobeli and Allen [26], Handler and Eisenhour [27] and von Wienskovski and Mönch [28].

In ellipsometry two parameters are measured corresponding to the relative phase change $(\Delta)$ and the relative amplitude ratio change $(\downarrow)$ of the two components of the polarized light wave, parallel with and perpendicular to the plane of incidence, upon reflection from a surface. The optical constants of $\mathrm{Ge}$ have becn calculated with the average $\Delta$ and $\psi$ values determined by the two-zone method in the photon-energy range from 1.5 to $3.5 \mathrm{eV}$. In fig. 4 they are compared with data from the literature [6,29]. The changes in th:se parameters (fig. $5 \delta \psi$ and fig. $6 \delta \Delta$ ) upon oxygen adsorption were recorded as a function of the photon-energy at which the ellipsometric measurements were performed, see also refs. [6-10,30-32]. We found optical transitions at 1.9, 2.3 and $3.0 \mathrm{eV}$ aiter cne monolayer of oxygen. At an oxygen coverage of 0.15 


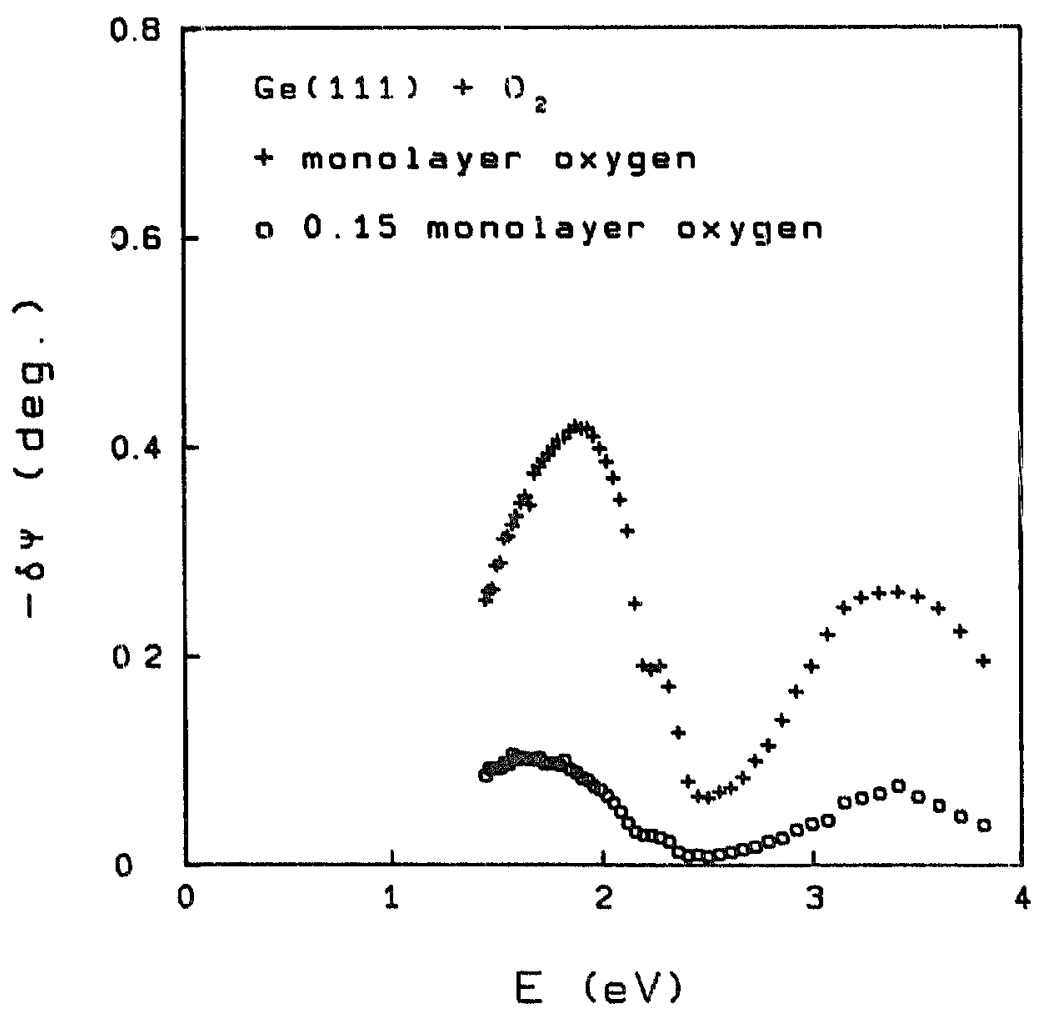

Fig. $5 . \delta \psi=\psi\left(\mathrm{O}_{2}\right)-\psi($ clean) as a function of photon energy.

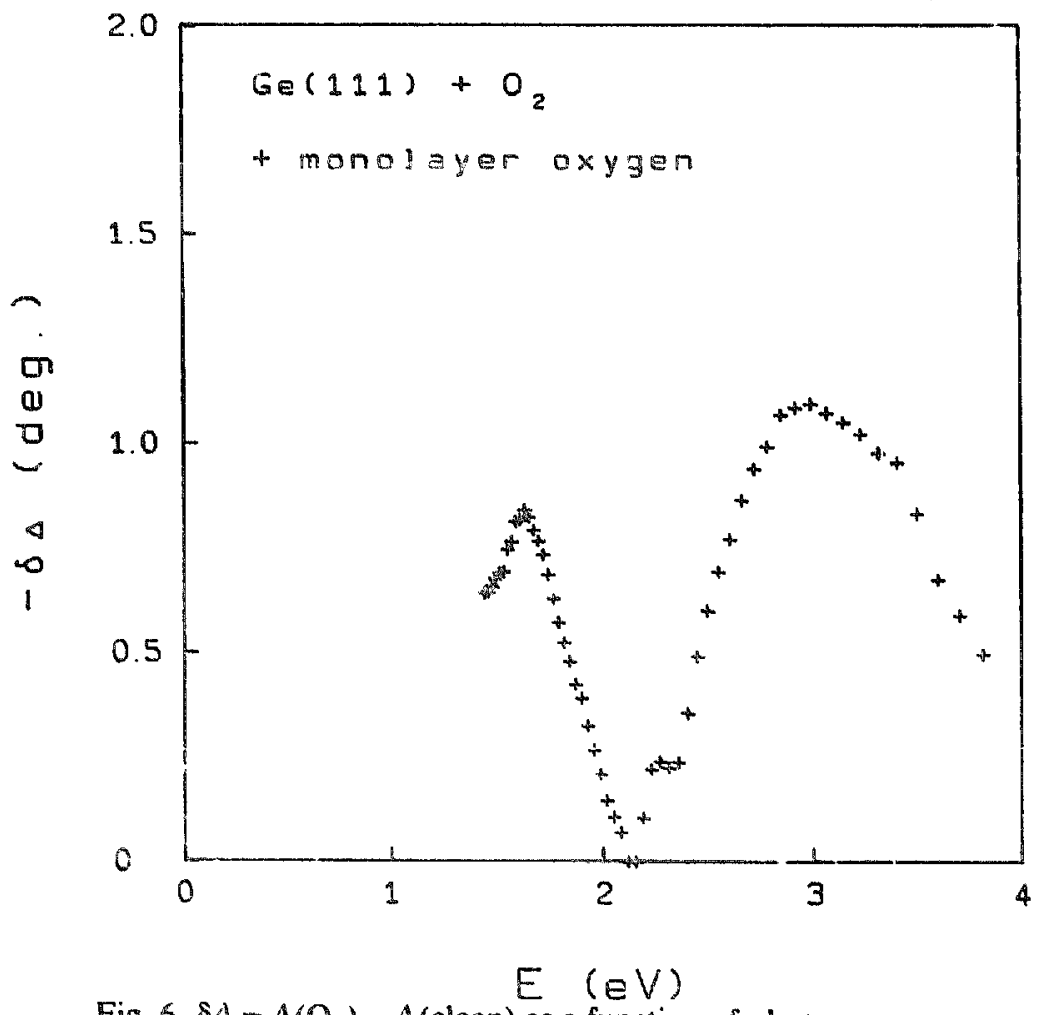

Fig. 6. $\delta \Delta=\Delta\left(\mathrm{O}_{2}\right)-\Delta$ (clean) as a function of phot on energy. 
monolayer (maximum surface conductivity) we found optical transitions at $1.7,2.3$ and $3.0 \mathrm{eV}$.

\subsection{Adsorption of oxygen on clean Ge(III) surfaces}

At different stages during oxygen adsorption subsequent pumping did not lead to significant changes in conductivity $[19,23,24,33,34]$, optical properties or oxygen coverage (Auger). Therefore the effect of oxygen adsorption may be called an irreversible effect. On the basis of Sun nev's calculations [16] we postulate that monolayer oxygen coverage (one oxygen atom per germanium surface atom) corresponds to $J\left(\mathrm{O}_{2} 510 \mathrm{eV}\right) / J(\mathrm{Ge} 1147 \mathrm{eV})=0.67$, where $J$ is the Auger peak to peak height.

Maximum surface conductivity is found for an oxygen coverage of 0.15 monolayer, at the same point Surnev [16] found a change in the elementary dipole moment with work function measurements.

A coverage of one monolayer oxygen was reached after an exposure of $1 \times 10^{6}-3 \times 10^{6} \mathrm{~L}$ in good accordance with Green and Liberman [35] and Boonstra [19]. According to Surnev [16] and Frantsuzov [36] the sticking coefficient decreases rapidly with the initially increasing oxygen coverage. We

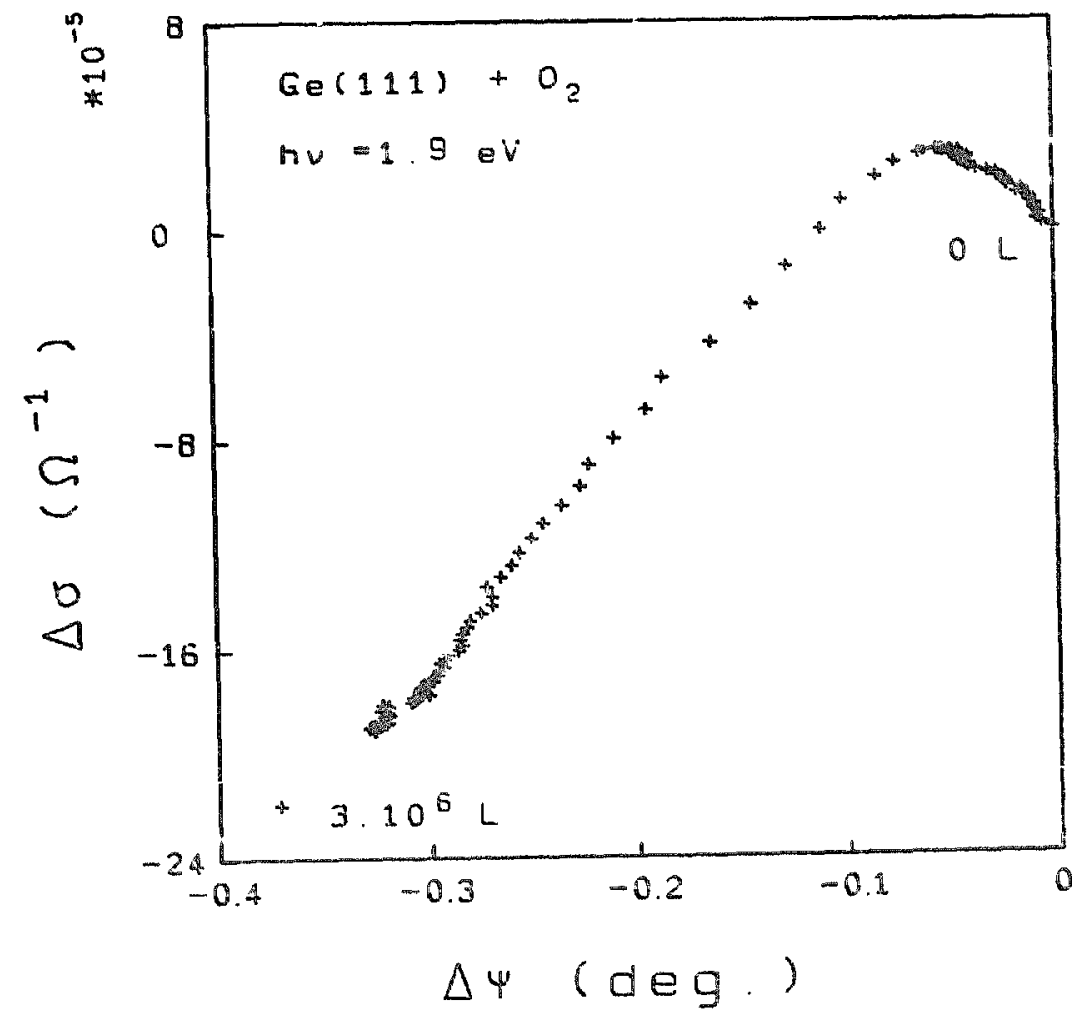

Fig. 7. $8 \psi$ versus conductivity changes for the optical transition at $1.9 \mathrm{eV}$. 
observed the same, but after several cleaning procedures and oxidations of the germanium surface we found a lower initial sticking coefficient compared with Surnev [16].

Ellipsometric measurements as a function of the photon energy at an oxygen coverage of 0.15 monolayer compared with one monolayer coverage measurements show no great difference in structure (see fig. 5) at 0.15 monolayer a peak at $1.7 \mathrm{eV}$ is shown and for monolayer coverage a peak at 1.9 $\mathrm{eV}$ is dominant. Due to overlap of the 1.7 and $1.9 \mathrm{eV}$ peaks, kinetic measurements give no extra information on absence of the $1.9 \mathrm{eV}$ peak below 0.15 monolayer.

During oxygen adsorption both $\delta \psi$ and the surface conductivity are fo!lowed continuously on the peaks at 1.7, 1.9, 2.3 and $3.0 \mathrm{eV}$. Plotting of $\delta \psi$ versus the change in surface conductivity results in a straight line with one kink occurring at an oxygen coverage of 0.15 monolayer (fig. 7). Within the limit of accuracy of the measurements all the peaks show the same behaviour, only the effect is most pronounced for the peak at $1.9 \mathrm{eV}$.

\section{Discussion}

The changes in surface conductivity are attributed to changes in the surface space charge layer, i.e. in the density of surface states. Because the condition of electrical neutrality must be satisfied we have:

$\sum-e N_{\mathrm{sa} i} f_{\mathrm{sa} i}: \sum e N_{\mathrm{sd} j}\left(1-f_{\mathrm{sd} j}\right)=e[\Delta N-\Delta P]$.

Field effect measurements have showr that $N_{\text {sa }}$ and $N_{\text {sd }}$ have values near the Fermi level in the order of at least $10^{13}-10^{14}$ per $\mathrm{cm}^{2}$. However the term in square brackets in eq. (5) has a value of about $7 \times 10^{11}$ per $\mathrm{cm}^{2}$ for a clean surface (band bending $-280 \mathrm{mv}$ ) and decreasing to dimosi eero during oxygen adsorption [19]. We must therefore conclude that both acceptor and donor surface states are present in very high densities on the $\mathrm{Ge}(111)$ surface.

We can only understand fig. 7 in our simple model if we assume that firstly more donor surface states disappear than acceptor surface states (this means $\delta_{\psi} \psi$ is lowering and $\sigma_{\mathrm{s}}$ is raising, see eq. (5)), followed by a second stage (after 0.15 monolayer of oxygen) where just more acceptor surface states disappear than donor surface states (this means $\sigma_{S}$ and $\delta \psi$ are both lowering). It might be possible that the peaks at 1.7 and $1.9 \mathrm{eV}$ are correlated to donor and acceptor surface states respectively. STM measurements of Ge(111)c(2 $\times 8)$ [43] indicate the presence of $1 / 4$ monolayer of adatoms. This may well explain any anomalies for uxygen coverages in this runge, perhaps a fraction of those adatoms are responsible for the first stage in the oxidation process.

The ellipsometric data gives only information on the energy gap between filled and unfilled states, we found optical transitions at 1.7, 1.9, 2.3 and 3.0 
$\mathrm{eV}$ above the energy gap of $\mathrm{Ge}(111) \mathrm{c}(2 \times 8)$. Nannarone et al. [11] measured the change in optical reflectivity for the $\mathrm{Ge}(111) 2 \times 1$ surface upon oxygen adsorption, the shape of their $\Delta R / R$ curve is almost the same as the shape of our $\delta \psi$ curve, they found transitions at 1.8 and $3.1 \mathrm{eV}$.

A Franz-Keldysh effect will appear in our experiments because of the change in surface electric field occurring during oxidation. Nannarone et al. $[11,37]$ mentioned that the change in reflectivity brought about by the oxide layer, cannot in principle be neglected. They say that such a term is very small except near the critical points of the bulk structure and in $\mathrm{Ge}$ it is negligible below $3.5 \mathrm{eV}$.

Meyer et al. [6,8] interpreted the change of $\psi$ for chemically adsorbed layers as being due to a substrate change upon chemical adsorption, i.e., the compensation of dangling bonds of the surface atoms. The substrate effect was described phenomenologically as the effective disappearance of a transition layer present at the clean surface. It was shown that the substrate effect increased linearly with the number of adsorbed nolecules [6] up to the saturation coverage where all dangling bonds had been compensated. The clean semiconductor surface is considered to consist of a bulk substrate covere $\AA$ with a surface states layer with thickness of $5 \AA$ with optical constants different from the buik (il car be shown that the choice of the layer thickness has no influence on the interpretation [8]). The effect of the thin oxide layer on the surface has been taken into account in both $\delta \psi$ and $\delta \Delta$, we have chosen an abritary thickness in our calculations of $1-3 \AA$. The relevant parameter for the transitions involving surface states is the differcnce between the imaginary part of the dielectric function of the surface states layer and that of the bulk at corresponding photon energies, i.e. $\operatorname{Im}\left(\varepsilon_{s s}-\varepsilon_{b}\right)$. The $\operatorname{Im}\left(\varepsilon_{s s}-\varepsilon_{h}\right)$ curve for $\mathrm{Ge}(111)$ as a function of the photon energy hy is given in fig. 8.

Bagchi, Barrera and Rajagopal [38,39] considered the problem of reflection fom a metal surface allowing for the nonlocality of the dielectric function in the direction normal to the surface, while assuming infinite periodicity parallel to the surface. The only assumption of their work was that the dielectric tensor of the material be diagonal for $k_{\|} \ll k$ (Fermi), and isotropic in the bu: work has been extended to semiconductor surfaces including off-diagonal components of the surface dielectric function by Del Sole et al. [40,41]. Following the work of Bagchi et al. we introduse:

$\Delta \varepsilon_{\mu, \mu}=\varepsilon_{\mu \mu \mu}-\varepsilon_{q u, u}^{0}, \quad \mu=x, y, z$,

$A_{v}(\omega)=\int d z \int d z\left[\Delta \varepsilon_{v, r}\right], \quad y=x, y$,

$\Lambda_{z}(\omega)=\int \mathrm{d} z \int \mathrm{d} z^{\prime}\left[1 / \Delta \varepsilon_{z z}\right]$,

where $\epsilon^{0}$ is the Fresnel solution. 


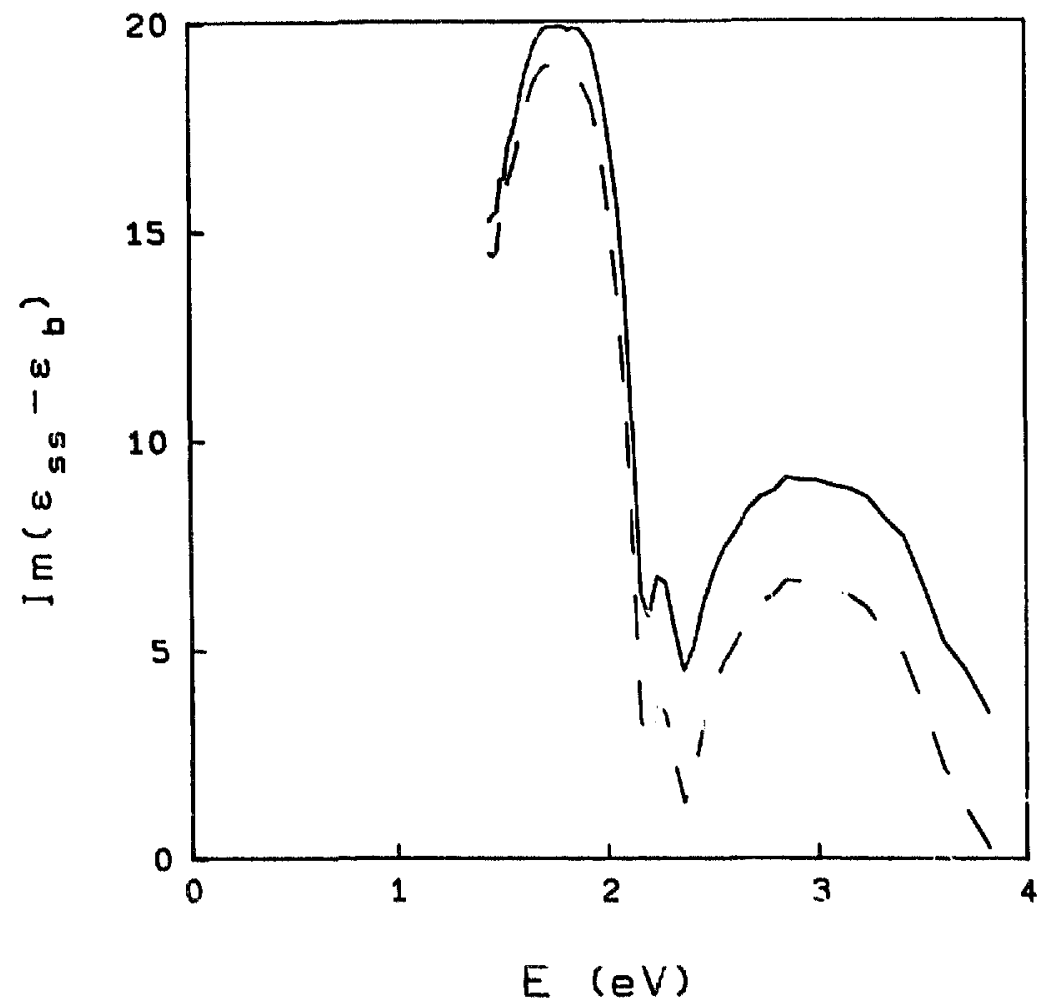

Fig. 8. The imaginary part of the difference in complex dielectric cor stant $\operatorname{Im}\left(\epsilon_{s s}-\epsilon_{b}\right)$ between the surface states layer and the bult int: trate for the clean $\mathrm{Ge}(111)$ surface as a function of the photon energy. Solid curve: without oxygen correction, dashed curve: influence of $1 \AA$ oxygen taken into account.

The ellipsometric effect induced by the removal of the surface states and oxide layer is contained in the expressions (7) and (8). From our measurements we can derive $\left(\epsilon_{\mathrm{b}} \Lambda_{z}+\Lambda_{x}\right)$ as a function of the photon energy (surface dielectric response). The imaginary part of $\epsilon_{\mathrm{s}}=\epsilon_{\mathrm{b}} \Lambda_{z}+\Lambda_{x}$ exhibits the same peaks as in $\delta \psi$, only the peak at $1.9 \mathrm{eV}$ is more pronounced (see fig. 9).

Our ellipsometric measurements as a function of the oxygen exposure (see fig. 5) indicated that there is no pronounced difference in the optical spectra between oxygen coverages below and above 0.15 morolayer. So it is unlikely that the significant difference in the adsorption at oxygen coverages lower and higher than 0.15 monolayer can be aitributed to the influence of the surface defects on the adsorption process.

Angle-resolved photoemission measurements $[3,4]$ showed the existence of two nondispersive peaks at encrgies of $0.8 \mathrm{eV}$ and $1.4 \mathrm{eV}$ below the Fermi level. The two features nearly disappeared upon hydrogenation of the reconstructed surface On the basis of this information, Bringans and Höchst [4] assigned these features to surface state emission. Inverse photoemission (Bremsstrahlung spectroscopy) would yield the energetic position of the empty 


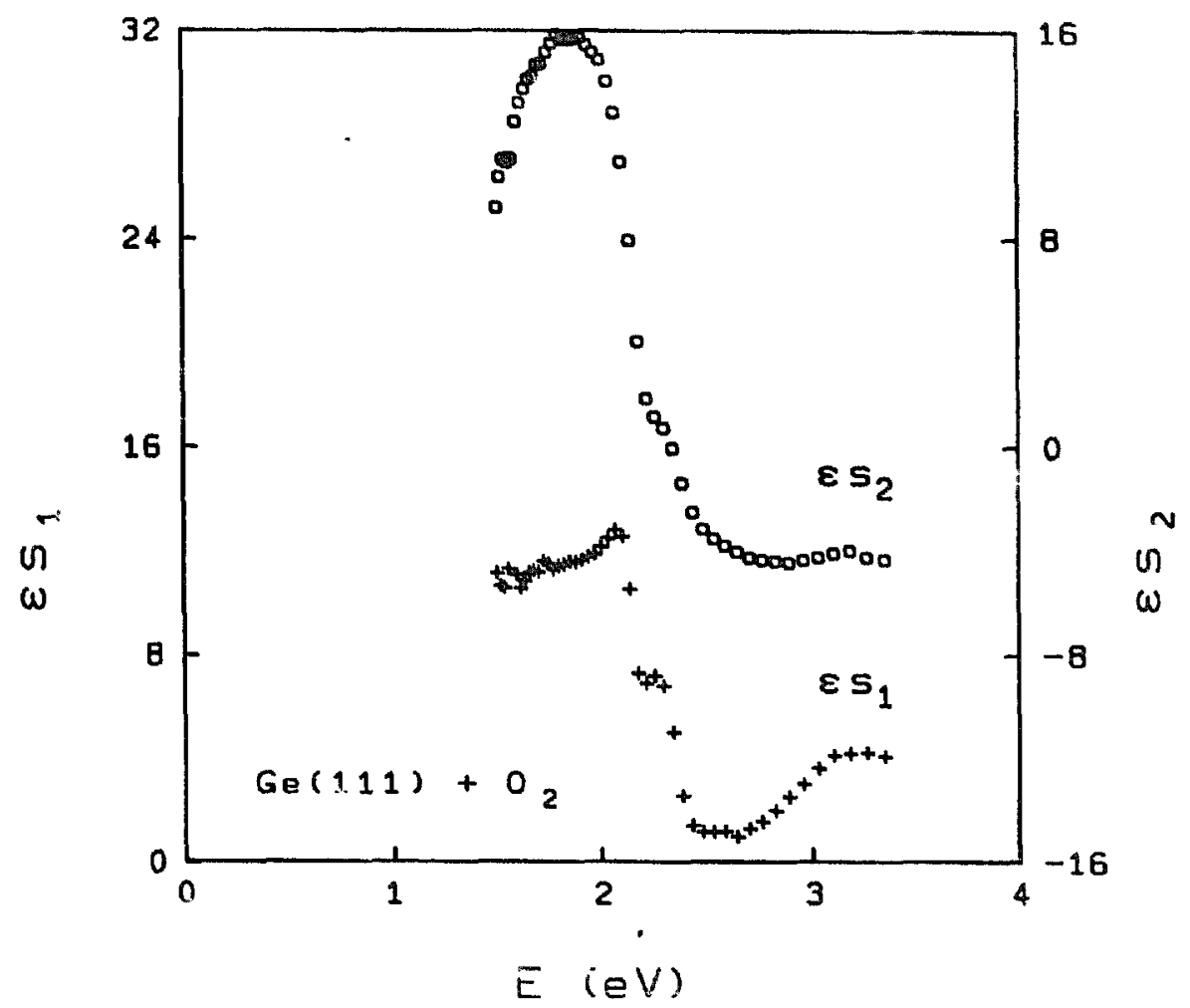

Fig. 9. Surface dielectric swsponse $\epsilon_{\mathrm{s}}=\epsilon_{\mathrm{b}} \Lambda_{z}+\Lambda_{x}$ calculated from the work of Bagchi et al. [39].

dangling bond surface states, but these data are not present at the moment. The surface band st:ucture of the $\operatorname{Ge}(111) \mathrm{c}(? \times 8)$ has not been calculated because of its large init cell

On the basis of cur uptical measurements and the photormission results [3-5] we suggest a possible surface state distribution around the Fermi level. If we assume an unitiled surface state lying about $0.9 \mathrm{eV}$ above the Fermi level, our peaks at 1.7 and $2.3 \mathrm{eV}$ can be explained. A surface state just below the Fermi level is recenily found by Aarts et al. [42], this surface state would explain the high density of surface states near the Fermi level (field effect measurements). The peaks at 1.0 and $3.0 \mathrm{eV}$ can be attributed to optical transitions from the known filled surface states to unfilled surface states in the conduction band.

\section{Acksuowledgenaents}

The authors wish to thank Professor M.J. Sparnaay for many stimulating discussions and J.G. Broeze for developing the ficid effect arrangement. 


\section{References}

[1] M.A. Olmstead, Surface Sci. Rept. 6 (1987) 159.

[2] F.J. Himpsel, Physica B117/118 (1983) 767.

[3] A.L. Wachs, T. Miller, T.C. Hsieh, A.P. Shapiro and T.C. Chiang, Phys. Rev. B32 (1985) 2326.

[4] R.D. Bringans and H. Höchst, Phys. Rev. B25 (1982) 1081.

[5] R.D. Bringans, R.I.G. Uhrberg and R.Z. Bachrach, Phys. Rev. B34 (1986) 2373.

[6] F. Meyer, E.E. de Kluizenaar and G.A. Bootsma, Surface Sci. 27 (1971) 88.

[7] F. Meyer and J.J. Vrakking, Surface Sci. 38 (1973) 275.

[8] F. Meyer, Phys. Rev. B9 (1974) 3622.

[9] F. Meyer and A. Kroes, Surface Sci. 47 (1975) 124.

[10] F. Meyer, Surface Sci. 56 (1976) 37.

[11] S. Nannarone, R. Chiaradia, F. Ciccacci, R. Memeo, P. Sassaroli, S. Selci and G. Chiarotti, Solid State Commun. 33 (1980) 593.

[12] E.G. Keim, L. Wolterbeek and A. van Silfhout, Surface Sci. 180 (1987) 565.

[13] A.H.M. Holtslag, Thesis, Twente University of Technology (1986).

[14] R.P. Tijburg, G.L. Dinghs and T. van Dongen, in: Vol. 79 of Institute of Physics Conference series, Karuizawa, Japan, 1985 (Institute of Physics, Reading, UK, 1985) ch. 6, p. 355.

[15] J.W.D. Martens, W.F. van den Bogert and A. van Silfhout, Surface Sci. 105 (1981) 275.

[16] L. Surnev, Surface Sci. 111 (1981) 364.

[17] F.J. Himpsel, D.E. Eastman, P. Heimann and B. Reihl, Phys. Rev. B24 (1981) 1120.

[18] G. Quentel and R. Kern, Surface Sci. 135 (1983) 325.

[19] A.H. Boonstra, Thesis, Eindhoven technical University (1967), Philips Res. Rept. 3 (1968).

[20] F. Meyer and M.J. Sparnaay, in: Surface Physics of Phosphors and Semiconductors, Eds. C.G. Scott and C.E. Reed (Academic Press, London, 1975) ch. 6.

[21] A. Many, Y. Goldstein and N.B. Grover, Semiconductor Surfaces (North-Holland, Amsterdam, 1965).

[22] D.R. Frankl, Electrical Properties of Semiconducter Surfaces (Pergamon, Oxford, 1967).

[23] M.J. Sparnaay and J. van Ruler, Physica 27 (1961) 153.

[24] M.J. Sparnaay, A.H. Boonstra and J. van Ruler, Surface Sci. 2 (1964) 56.

[25] R. Sciwatz and M. Green, I. Appl. Phys. 29 (1958) 1034.

[26] G.W. Gobeli and F.G. Allen, Surface Sci. 2 (1964) 402.

[27] P. Harulier and S. Eisenhour, Surface Sci. 2 (1964) 64.

[28] J. von Wienskovski and W. Mönch, Phys. Status Solidi 45 (1971) 583.

[29] D.E. Aspnes and A.A. Studna, Surface Sci. 96 (1980) 294.

[30] R. Dorn and H. Lüth, Phys. Rev. Letters 33 (1974) 1024.

[31] R. Dorn, H. Lüth and H. Ibach, Surface Sci. 42 (1974) 583.

[32] F. Meyer, Surface Sci. 56 (1976) 37.

[33] A.H. Boonstra, J. van Ruler and M.J. Sparnaay, Proc. Konink. Ned. Akad. Wetenschap. B66 (1953) 70.

[34] A.H. Boonstra, J. van Ruler and M.J. Sparnaay, Proc. Konink. Ned. Akad. Wetenschap. B66 (1963) 64.

[35] M. Green and A. Libeiman, J. Phys. Chem. Solids 23 (1962) 1407.

[36] A.A. Flantsuzov and N.I. Makrushin, Surface Sci. 40 (1973) 320.

[37] F. Ciccacci, S. Selci, G. Chiarotti, P. Chiaradia and A. Cricenti, Surface Sci. 168 (1986) 28.

[38] R.G. Barrera and A. Bagchi, Phys. Rev. B24 (1981) 1612.

[39] A. Bagchi, R.G Barrera and A.K. Rajagopal, Phys. Rev. B20 (1979) 4824.

[40] R. Del Sole and A. Selloni, Phys. Rev. B30 (1984) 883.

[41] R. Del Sole and E. Fiorino, Phys. Rev. R29 (1984) 1531.

[42] J. Aarts, A.J. Hoeven and P.K. Larsen, to be published.

[43] R.S. Becker, J.A. Golovchenko and B.S. Swartzentruber, Phys. Rev. Letters 54 (1985) 2678. 